\title{
Automatic coil selection for channel reduction in SENSE-based parallel imaging
}

\author{
Mariya Doneva · Peter Börnert
}

Published online: 3 June 2008

(C) ESMRMB 2008

\section{Erratum to: Magn Reson Mater Phy DOI 10.1007/s10334-008-0110-x}

The original version of this article unfortunately contained errors in Figs. 6 and 7. The correct figures are given below.
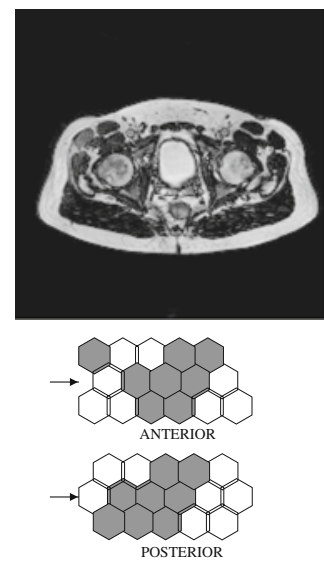

(a)
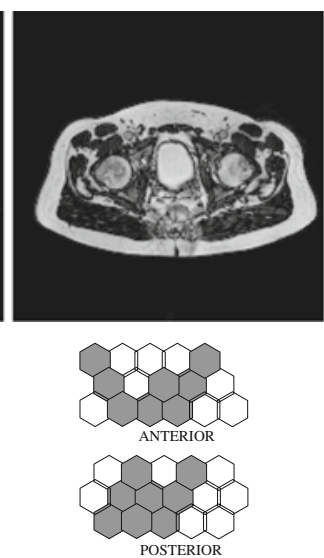

(b)
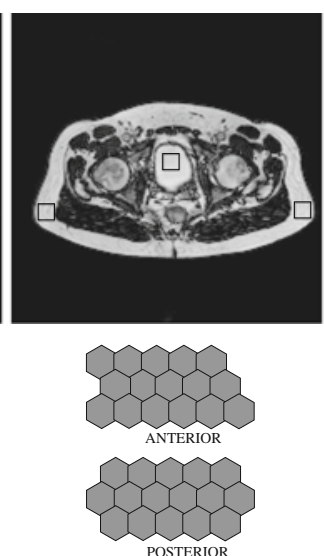

(c)
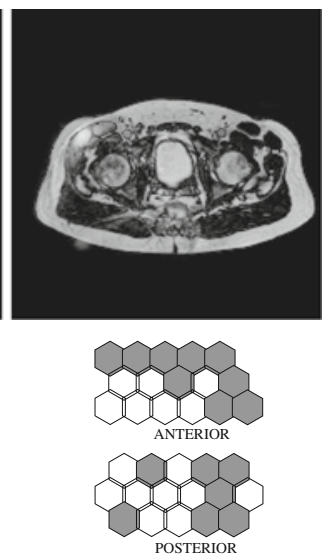

(d)

Fig. 6 In vivo results (16 out of 32). A transversal slice extracted from 3D data reconstructed with a 16 coils selected with the SVD-based coil selection algorithm, b 16 coils selected with the SNR-based coil selection algorithm, $\mathbf{c}$ the full set of 32 coils, d 16 coils rejected by the SVD coil selection algorithm. The selected coil elements and the slice position are marked on the coil array geometry diagrams. The

two algorithms show only small differences in the coil selection. The resulting images $(\mathbf{a}, \mathbf{b})$ are similar and do not show a visible decrease in SNR compared to the full coil set image (c). The image of the SVD rejected coils shows residual aliasing artifacts (d). The regions used for SNR estimation in this slice are indicated on the image (c)

The online version of the original article can be found under doi:10.1007/s10334-008-0110-x.

\section{Doneva}

University of Oldenburg, Oldenburg, Germany

e-mail: mariya.doneva@philips.com

\section{P. Börnert (凶)}

Philips Research Laboratories, Röntgenstraße 24-26,

23335 Hamburg, Germany

e-mail: peter.boernert@philips.com 

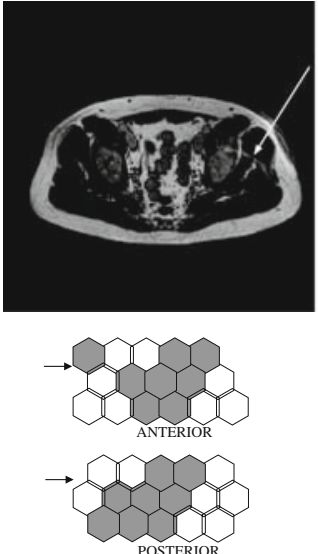

(a)
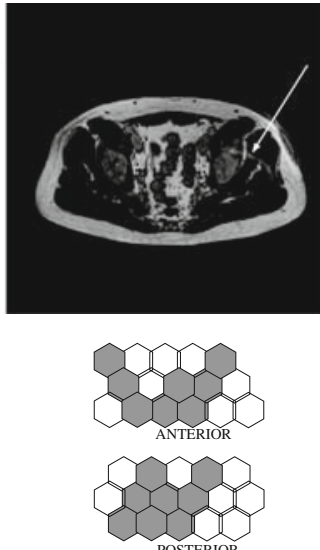

(b)
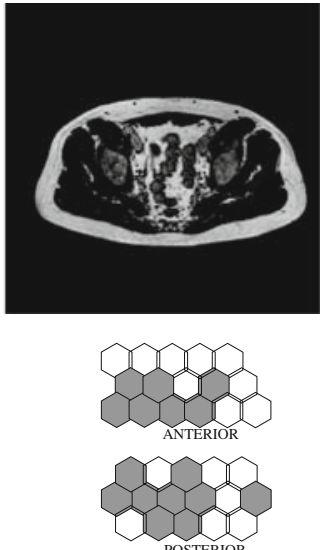

(c)
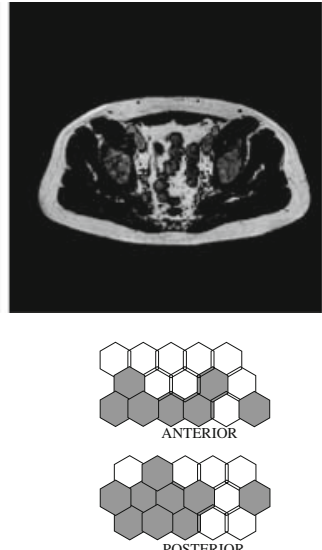

(d)

Fig. 7 In vivo results (16 out of 32). A transversal slice extracted from the same 3D data shown in Fig. 6 at different slice position. Images, reconstructed with (a) 16 coils selected with the SVD-based coil selection algorithm (b) 16 coils selected with the SNR-based coil selection

algorithm show residual fold-over artifacts (indicated by the arrow) The same slice, reconstructed with the 16 coils, selected using local optimization (c) using the SVD-based coil selection and (d) using the SNR-based coil selection show improved image quality 